\title{
TV-Talkshows und ihr authentisches Potenzial für den Russischunterricht
}

\author{
Magdalena Kaltseis (Innsbruck)
}

Автор Аанной статьи анализирует потенциал российских ток-шоу и Аемонстрирует возможности их использования в преподавании РКИ. Работа с таким видом телепрограмм способствует развитию не только языковых и культурологических компетенций, но и так называемой аудиовизуальной компетенции. Телевидение является до сих пор неотьемлемой частью повседневной российской культуры, а ток-шоу - одним из самых популярных видов телепрограмм. Аутентичность, непродолжительность, многообразие и развцечение - это Аишь некоторые преимущества ток-шоу. Ввиду этого, использование Аанного телевизионного жанра необходимо Аля разнообразия в классе и Аля развития знаний и критических суждений о российском телевидении в целом.

\section{Einleitung}

Im Deutschen erinnern Enunziationen wie Lass sie reden oder Die Zeit wird's zeigen an Zeilen aus Songtexten oder an Romantitel. Tatsächlich sind ihre russischen Pendants - Pust' govorjat und Vremja pokažet-jedoch die Namen bekannter TVTalkshows in Russland. Ein Blick auf das Programm des Ersten Kanals (Pervyj kanal) genügt, um zu erkennen, dass RussInnen rund um die Uhr mit Talkshows - Gesprächssendungen - versorgt werden. Nur in wenigen Zeitschienen, den sogenannten „Slots“ (Semeria 2002: 161), finden sich keine Talkshows. Diese „Slots“ sind gefüllt mit Nachrichten, Spielfilmen, Gameshows oder Serien. In allen anderen Zeitabschnitten wird getalkt, gestritten, lautstark diskutiert, geschimpft oder geschrien. Obwohl aufgrund ihrer Anzahl zunächst der Anschein 
entstehen könnte, dass Talkshows schon immer ein fester Bestandteil der russischen Fernsehwelt waren, sind diese ein Phänomen der Post-Perestrojka-Periode.

Im Westen existieren Talkshows seit den 1950er-Jahren und zählen somit zu jenen Formaten, die quasi von Beginn an im Medium Fernsehen präsent waren. Allgemein definieren sich Talkshows durch die Anwesenheit eines oder mehrerer ModeratorInnen (hosts), die sich mit einem oder mehreren Gästen über ein beliebiges Thema unterhalten. Konstitutiv für dieses Genre ist die Präsenz „eines sich parasozial am Gespräch beteiligenden Fernsehpublikums" (Tenscher \& Schicha 2002: 11). Im sowjetischen Russland glänzte die Talkshow durch ihre Abwesenheit im Fernsehprogramm, da sie von der Sowjetregierung aufgrund der Missachtung der schriftlichen Sprache und ihrer verbalen Freizügigkeit als gefährlich eingestuft wurde (vgl. Hutchings \& Rulyova 2009: 89). Live-Talk oder freier Talk existierte nur in Form eines verlängerten Interviews mit Personen aus dem Kulturbereich und hatte keine Gemeinsamkeit mit spontanen Gesprächen westlicher Talkshows. Nach dem Zerfall der Sowjetunion und unter dem Schlagwort der glasnost' veränderte sich jedoch das Fernsehen in Russland. Dadurch konnte sich die Talkshow, die als unflektierbare tok-šou in den Wortschatz aufgenommen wurde, dank ihrer niedrigen Produktionskosten, ihres hohen Massenanreizes und der freien Rede auch in Russland etablieren (vgl. ebd.: 90).

In den letzten Jahren hat die Anzahl von Talkshows im russischen Fernsehen stark zugenommen (vgl. Zvereva 2012: 84), und es gibt eine breite Palette an Themengebieten, die abgedeckt wird. Talkshows sind jedoch kein kurzfristiges Phänomen einer sich im Wandel befindenden Fernsehlandschaft, sondern ein „augenscheinlicher Indikator tiefer gehender kultureller, medialer und auch politischer Veränderungen“, die unsere Gesellschaft prägen (Tenscher \& Schicha 2002: 10). Sie sind sowohl „kultureller Mediator“ (Hutchings \& Rulyova 2009: 90) als auch ein „populärkulturelles Produkt“ (Semeria 2002: 177), das der Gesellschaft zur Verständigung über sich selbst dient (vgl. Mikos 1994: 197). Als Teil der Populärkultur und Indikator aktueller Veränderungen sollten Talkshows daher auch im Russischunterricht nicht ignoriert werden, da sie über ein hohes didaktisches sowie authentisches Potenzial verfügen, welches im Unterricht sowohl zur Förderung der sprachlichen Kompetenz als auch der Interkulturalität genutzt werden kann. Im vorliegenden Artikel werden deshalb Beispiele dafür

Anka Bergmann, Olga Caspers \& Wolfgang Stadler (Hg.)

Didaktik der slawischen Sprachen - Beiträge zum 1. Arbeitskreis in Berlin (12.-14.9.2016)

(C) 2018 innsbruck university press, ISBN 978-3-903187-11-5, DOI 10.15203/3187-11-5 
gegeben, wie TV-Talkshows in den Russischunterricht integriert werden können.

\section{Zum Forschungsstand: Authentizität und audiovisuelles Material im Fremdsprachenunterricht}

Die Forderung nach echten, authentischen Texten im Fremdsprachenunterricht (FSU) existiert bereits seit Beginn der Kommunikativen Wende Anfang der 1970er Jahre: „Communicative language teaching (CLT) promoted a preference for real or authentic texts, that is, texts which have been produced by and/or for expert users of the language for use outside of the classroom [...]" (Roberts \& Cooke 2009: 622). In enger Verbindung zu dem Begriff der Authentizität steht seit damals auch der vermehrte Einsatz (audio-)visueller Medien im FSU, da diese authentisches Quellenmaterial, ergo für MuttersprachlerInnen konzipierte Texte, bereitstellen. Inzwischen ist Authentizität im Zuge der Publikation des Gemeinsamen europäischen Referenzrahmens für Sprachen (GeR 2001), der Einführung von Bildungsstandards (in Deutschland: 2004/5; in Österreich: 2008) und der Kompetenz-, Handlungs- und Aufgabenorientierung sowie der Mehrsprachigkeitsdidaktik und der zunehmenden Auseinandersetzung mit Lernerautonomie zu einem Standardkriterium im modernen FSU avanciert. Authentizität im Fremdsprachenunterricht bedeutet allgemein, dass die im Unterricht verwendete Sprache so weit wie möglich dem echten Sprachgebrauch außerhalb des Unterrichts entsprechen soll (vgl. Thaler 2017: 16). Trotz dieser relativ einfachen Beschreibung Thalers der Bedeutung der Authentizität für den FSU gibt es in der Forschungsliteratur keinen Konsens zur Frage, was einen authentischen FSU auszeichnet. Während einige AutorInnen eine Konzentration auf authentische Lernmaterialien fordern (vgl. Al Azri \& Al-Rashdi 2014, Crossley et al. 2007, Mac 2011), plädieren andere wiederum für authentische Aufgabenstellungen unter Einbindung der Lernenden (vgl. Arnold 1991, Mishan 2005, Nosonovič \& Mil'rud 1999, Roberts \& Cooke 2009). Die Autentizität, die sich auf die im FSU verwendeten Materialien bzw. Texte bezieht, wird auch als Material- bzw. Textauthentizität bezeichnet. Im Unterschied dazu existieren

Anka Bergmann, Olga Caspers \& Wolfgang Stadler (Hg.)

Didaktik der slawischen Sprachen - Beiträge zum 1. Arbeitskreis in Berlin (12.-14.9.2016)

(C) 2018 innsbruck university press, ISBN 978-3-903187-11-5, DOI 10.15203/3187-11-5 
die Begriffe der Situations- und der Interaktionsauthentizität. Erstere geht davon aus, dass die Lernenden die Aufgaben bzw. die Situationen, mit denen sie im Unterricht konfrontiert werden, als authentisch empfinden sollen. Ebenso wird mit der Interaktionsauthentizität ausgedrückt, dass Authentizität nicht vom verwendeten Material abhängt, sondern von der authentischen Lernsituation, in der die Lernenden selbst kommunikativ handeln und interagieren (vgl. Bechtel \& Roviró 2010: 208f.). Arnold (1991: 238) bringt diesen Dissens in der Literatur mit folgender Aussage gut auf den Punkt: "Use of authentic materials does not imply that the tasks will be authentic." Folglich setzt sich Authentizität im FSU nicht allein aus dem im Unterricht verwendeten Material zusammen, sondern auch aus der Art und Weise, wie dieses im Unterricht eingesetzt wird und welche Aufgabenstellungen dafür konzipiert werden.

Authentisches Material für den FSU ist heute dank des Internets leicht zugänglich und ohne größere Probleme zu finden. Das betrifft u. a. Zeitungstexte, die online publiziert werden, Blogs, soziale Medien sowie Videomaterial und Filme auf Internetseiten wie YouTube. Insb. audiovisuelles Material, wie Videoclips, (Kurz-/ Spiel-)Filme, Werbefilme, Fernsehshows oder Nachrichtensendungen, bieten aufgrund der Kombination von Bild und Ton sowie ihrer Inter- und Multimodalität vielseitige Einsatz- und Analysemöglichkeiten im FSU. Wie zahlreiche Untersuchungen zeigen (vgl. z. B. Brook 2011, Herron et al. 2006, Lay 2009, Lommel, Laenen \& Ydewalle 2006, Rokni \& Ataee 2014, Scherpinski 2014), eignen sich audiovisuelle Medien nicht nur aufgrund ihrer Vielseitigkeit, ihrer Lebendigkeit, ihrem hohen Motivationspotenzial und ihrer Authentizität für den Unterricht, sondern auch, weil die Lernenden bei der Verwendung audiovisueller Unterrichtsmaterialien bessere Verstehensleistungen erzielen als bei rein auditiven (vgl. Scherpinski 2014: 178) bzw. textbasierten Materialien (vgl. Herron et al. 2006). Thaler (2007: 12) fasst die Vorteile des Hör-Seh-Verstehens wie folgt treffend zusammen: „Gesellt sich zum Hören noch das Sehen, kommt zur authentischen Sprache die beobachtbare Handlung dazu, paralinguistische Handlungsmerkmale werden sichtbar, aus den disembodied voices werden beobachtbare native speakers, landeskundliche Elemente werden seh-/einseh-/verstehbar, Bilder können genauere Informationen liefern, visuelle Markierungen fungieren als Erinnerungshilfe, der Weltausschnitt erweitert sich, Lernen wird einsichtiger und motivierender [...]".

Anka Bergmann, Olga Caspers \& Wolfgang Stadler (Hg.)

Didaktik der slawischen Sprachen - Beiträge zum 1. Arbeitskreis in Berlin (12.-14.9.2016)

(C) 2018 innsbruck university press, ISBN 978-3-903187-11-5, DOI 10.15203/3187-11-5 
Wie aus diesem Zitat Thalers hervorgeht, leisten audiovisuelle Medien einen wichtigen Beitrag für das interkulturelle Lernen, da mithilfe von bewegten Bildern auch die Kultur und Spezifika des jeweiligen Ziellandes einfach vermittelt werden können (vgl. hier auch Caspers \& Scharlaj im vorliegenden Sammelband). ${ }^{1}$ Ein weiteres Potenzial audiovisueller Medien liegt in ihrer Rezeption, da diese dem natürlichen Konsum und Verstehen dieser Medien entspricht (vgl. Scherpinski 2014: 171). Zudem wird mithilfe von bewegten Bildern auch „Paraverbales, Extraverbales und Nonverbales" sichtbar (ebd.: 173) und Persönlichkeitsbildung sowie „affektive und imaginative Fähigkeiten“ werden mit ihrer Hilfe gefördert (García 2016: 340).

Wie anhand der angeführten Literatur erkennbar wird, findet in der Fremdsprachenforschung eine intensive Beschäftigung mit dem Potenzial audiovisueller Medien für den FSU, v. a. mit Filmen jeglicher Form, statt. Mit dem Aufkommen neuer technischer Möglichkeiten in Bildungseinrichtungen hat sich die Filmdidaktik in den letzten Jahren kontinuierlich weiterentwickelt und zahlreiche Publikationen widmen sich den Möglichkeiten und Methoden des Einsatzes von Filmen im FSU, allen voran in der Anglistik (vgl. z. B. Abraham 2012, Henseler, Möller \& Surkamp 2011, Lütge 2012, Sherman 2003, Thaler 2014). Auch für den Russischunterricht existieren bereits Vorschläge dafür, wie Spiel-, Trick- oder Kurzfilme in den Unterricht integriert werden können (vgl. z. B. Bertram 2011, Binder \& Kaltseis 2016a, 2016b, Engel \& Bacher 2011, Kaltseis 2015, Laschet 2008, Nadchuk \& Behr 2012, Vakal 2005, Zühlke 2007). Allerdings wird an der bisherigen Filmdidaktik auch Kritik aufgrund ihrer Einseitigkeit geübt, da sich diese hauptsächlich auf Sprach- und Tonverständnisaufgaben, ergo auf reine Hörverständnisaufgaben, die durch bewegte Bilder vereinfacht werden, konzentriere. Dadurch werden häufig die visuelle Komponente sowie die Filmästhetik vernachlässigt und auch Beobachtungsaufgaben oder die Interpretation von Filmbildern finden wenig Berücksichtigung (vgl. García 2016: 340, Satzinger 2016: 246). In diesem Zusammenhang ist der Terminus der visual

1 An dieser Stelle muss jedoch betont werden, dass Spielfilme oder anderes filmisches Material nicht als einzige Informationsquelle für landeskundliche Informationen dienen sollten, weil die filmische Welt fiktiv bzw. subjektiv ist und niemals die Realität in ihrer Gesamtheit abbildet.

Anka Bergmann, Olga Caspers \& Wolfgang Stadler (Hg.)

Didaktik der slawischen Sprachen - Beiträge zum 1. Arbeitskreis in Berlin (12.-14.9.2016)

(C) 2018 innsbruck university press, ISBN 978-3-903187-11-5, DOI 10.15203/3187-11-5 
literacy oder der „audiovisuelle[n] Kompetenz (audio-visual literacy)“ zu nennen, die das übergeordnete Ziel des Einsatzes audiovisueller Medien im FSU darstellt, da neben dem Hör-Seh-Verstehen die Fähigkeit der Filmanalyse, das interkulturelle Lernen sowie Methoden-Kompetenz geschult werden sollen (Thaler 2010: 142f.). In Bezug auf das Fernsehen existiert seit einigen Jahren im FSU der Begriff der textübergreifenden Fernsehkompetenz (TV literacy), welche die „Fähigkeit [beschreibt], sich kritisch mit den Formen, Funktionen und Intentionen, die hinter der heutigen `Fernsehkultur stecken, auseinanderzusetzen" (Surkamp 2017: 351). Ein allgemeines Ziel der Verwendung von audiovisuellem Material stellt somit nicht nur die Fähigkeit dar, Bilder lesen, verstehen und interpretieren zu lernen (vgl. Abraham 2012: 27), sondern bezüglich des Fernsehens auch die Kompetenz, dieses Medium repräsentationskritisch und kulturwissenschaftlich zu hinterfragen (vgl. Surkamp 2017: 350).

Aus diesem Grund beschäftigt sich der vorliegende Aufsatz mit der Frage, wie neben dem Hör-Seh-Verstehen auch die interkulturelle Kompetenz und das Film-/Fernsehverstehen besser gefördert und in den Russischunterricht integriert werden können. Als Beispiel dafür werden russische TV-Talkshows vorgestellt, da diese, wie auch das russische Fernsehen allgemein, in der Literatur bisher noch kaum berücksichtigt werden. Für den Russischunterricht stellen Fernsehtalkshows jedoch aufgrund ihrer spezifischen Themengebiete und ihrer Vielseitigkeit ein besonders buntes und breit einsetzbares Genre dar.

\section{Kritische Überlegungen zu Fernsehen und Talkshows in Russland}

Wie in Abschnitt 2 illustriert, existieren zahlreiche Vorschläge, wie Trick-, Spieloder Kurzfilme in den Russischunterricht integriert werden können. Beispiele für die Integration des russischen Fernsehens im Unterricht dagegen sind kaum vorhanden und somit fristet das russische Fernsehen mit seinen Dokumentationen, Serien und Shows ein eher stiefmütterliches Dasein. Im universitären Russischunterricht werden zwar häufig russische Nachrichtensendungen behandelt; diese sind jedoch auch auf höheren Niveaustufen noch schwer verständlich,

Anka Bergmann, Olga Caspers \& Wolfgang Stadler (Hg.)

Didaktik der slawischen Sprachen - Beiträge zum 1. Arbeitskreis in Berlin (12.-14.9.2016)

(C) 2018 innsbruck university press, ISBN 978-3-903187-11-5, DOI 10.15203/3187-11-5 
da sehr schnell gesprochen wird und bestimmte grammatische Strukturen, wie Partizipien, vermehrt verwendet werden. Ein weiteres Problem des russischen Fernsehens stellt nicht zuletzt seit der Ukrainekrise 2014 der Missbrauch dieses Mediums als Propagandainstrument des Kremls dar. Informationen, die über das russische Staatsfernsehen verbreitet werden, sollten daher niemals unreflektiert übernommen werden. Gleichzeitig besteht darin aber auch eines der Potenziale in der Auseinandersetzung mit dem russischen Fernsehen, da sie die Möglichkeit bietet, die Lernenden zu sensibilisieren, sie auf die Art und Weise der Informationsvermittlung im russischen Fernsehen bzw. in den Nachrichten aufmerksam zu machen und diese mit der Berichterstattung in westlichen Ländern zu vergleichen.

Für die Eingliederung des russischen Fernsehens in den Unterricht spricht in erster Linie, dass dieses trotz steigender Popularität des Internets nach wie vor das beliebteste und ebenso das einflussreichste Medium in Russland ist (vgl. Zvereva 2012: 10). Die Tatsache, dass auch russische MuttersprachlerInnen das Medium Fernsehen intensiv konsumieren, macht es für Sprachlernende äußerst authentisch. Eines der am häufigsten gesehenen Fernsehformate ist die Talkshow. Das zeigt, wie o. g., nicht nur ein Blick auf das tägliche Fernsehprogramm, in dem Talkshows zahlreich vertreten sind, sondern das demonstrieren auch die wöchentlichen Erhebungen des Markt- und Meinungsforschungsinstituts TNSRussland. Eine erst kürzlich erschienene Umfrage des Levada-Zentrums zur russischen Medienlandschaft bestätigt dieses Phänomen (vgl. Volkov \& Gončarov 2017). Aus dieser Studie des Levada-Zentrums geht deutlich hervor, dass ca. ein Drittel aller RussInnen politische Talkshows, wie bspw. Večer s Vladimirom Solovëvym, regelmäßig ansieht. Neben dem vermeintlichen Informationsgehalt wird an ihnen besonders ihr ,fesselnder' (zachvatyvajušč $i j)$ Charakter geschätzt.

Obwohl nicht-politische, unterhaltungsorientierte Talkshows in der hier angeführten Levada-Umfrage nicht vorkommen, kann aufgrund der wöchentlichen TNS-Erhebungen davon ausgegangen werden, dass sich nicht-politische Talkshows einer mind. genauso großen Beliebtheit erfreuen. Letztere eignen sich besonders für den Einsatz im Russischunterricht, da sie näher an der Lebenswelt der SchülerInnen und sprachlich sowie inhaltlich leichter verständlich sind als politische Talkshows.

Anka Bergmann, Olga Caspers \& Wolfgang Stadler (Hg.)

Didaktik der slawischen Sprachen - Beiträge zum 1. Arbeitskreis in Berlin (12.-14.9.2016)

(C) 2018 innsbruck university press, ISBN 978-3-903187-11-5, DOI 10.15203/3187-11-5 


\section{Das Potenzial russischer Talkshows für den Unterricht}

Die Idee, Talkshows als Medium im FSU zu nutzen, ist kein Novum. Sherman (2006: 82-90) erkannte bspw. bereits das Potenzial von Talkshows für den Fremdsprachenunterricht und stellte Vorschläge für ihren Einsatz im Unterricht vor. Die positiven Eigenschaften von Talkshows für das Sprachenlernen können in Anlehnung an Sherman (ebd.: 83f.) wie folgt zusammengefasst werden:

- Das Zentrum von Talkshows bildet das Gespräch - der Talk.

- Das Gespräch in Talkshows wird durch die Paarsequenz ,Frage-Antwort determiniert.

- Talkshows beziehen das Publikum mit ein.

- Talkshows haben ein bestimmtes Thema und wollen als Unterhaltungsprogramm die Aufmerksamkeit der ZuseherInnen erlangen. Dabei konzentrieren sie sich auf Themenbereiche, die großes Interesse hervorrufen, wie z. B. prominente Personen, wichtige Ereignisse oder Streitfragen.

- Talkshows stellen Personen, ihren Charakter, ihre Gefühle, ihren Kleidungsstil etc. in den Mittelpunkt.

Obwohl Talkshows auch Informationscharakter haben, geht es in ihnen in erster Linie um Unterhaltung. Nicht-politische Talkshows können thematisch grob in zwei Kategorien unterteilt werden: in die Promitalkshow und in die Bekenntnis-/ problemorientierte Talkshow (vgl. Creeber 2015: 194-199, Plake 1999: 32f.). Ebenso kann die Einteilung von Talkshows auf ihrer Sendezeit basieren: Morning Talkshows (6-10 Uhr), Daytime Talkshows (10-16 Uhr) und Late-NightTalkshows (ab 22:30 Uhr). Auch nicht-politische Talkshows in Russland können diesen Kategorien zugeordnet werden. In der nachfolgenden Tabelle (Tab. 1) werden nun einige ausgewählte Talkshows, die sich für den Russischunterricht eignen, angeführt.

Die in Tab. 1 präsentierten Talkshows stammen alle vom staatlichen Pervyj kanal. Auf diesem Sender sind besonders viele Talkshows zu finden und er weist in Russland neben dem Sender Rossija die höchste Reichweite auf. Außerdem sind die jeweiligen Ausgaben der Talkshows auf der Seite des Pervyj kanal on-

Anka Bergmann, Olga Caspers \& Wolfgang Stadler (Hg.)

Didaktik der slawischen Sprachen - Beiträge zum 1. Arbeitskreis in Berlin (12.-14.9.2016)

(C) 2018 innsbruck university press, ISBN 978-3-903187-11-5, DOI 10.15203/3187-11-5 
Tabelle 1: Liste möglicher Talkshows für den Russischunterricht auf dem Pervyj kanal

\begin{tabular}{|c|c|c|c|}
\hline Kategorie & Themen & Talkshows & Kurzbeschreibung \\
\hline $\begin{array}{l}\text { Problemorientierte } \\
\text { Daytime-Talkshow }\end{array}$ & $\begin{array}{l}\text { Mode \& } \\
\text { Lebensstil }\end{array}$ & $\begin{array}{l}\text { Modnyj } \\
\text { prigovor }\end{array}$ & $\begin{array}{l}\text { Mode-/Umstylingshow, in der } \\
\text { wie vor Gericht ein modisches } \\
\text { Urteil (modnyj prigovor) über den } \\
\text { Kleidungsstil einer Person von } \\
\text { Modeexperten und Verwandten/ } \\
\text { Freunden gefällt wird }\end{array}$ \\
\hline $\begin{array}{c}\text { Promitalk } \\
\text { Daytime-Talkshow }\end{array}$ & $\begin{array}{l}\text { Familie, } \\
\text { Karriere, } \\
\text { Erfolg etc. }\end{array}$ & $\begin{array}{l}\text { Poka vse } \\
\text { doma }\end{array}$ & $\begin{array}{l}\text { Tischgespräch mit russischen } \\
\text { Prominenten bei ihnen zuhause }\end{array}$ \\
\hline $\begin{array}{c}\text { Promitalk } \\
\text { Daytime-Talkshow }\end{array}$ & $\begin{array}{l}\text { Kulinarik, } \\
\text { Karriere, } \\
\text { Erfolg }\end{array}$ & Smak & $\begin{array}{l}\text { Kulinarische Unterhaltungs- } \\
\text { talkshow mit Ivan Urgant und } \\
\text { russischen Prominenten }\end{array}$ \\
\hline $\begin{array}{l}\text { Promitalk Late- } \\
\text { Night-Talkshow }\end{array}$ & $\begin{array}{l}\text { Comedy, } \\
\text { aktuelle } \\
\text { Ereignisse } \\
\text { etc. }\end{array}$ & $\begin{array}{l}\text { Večernij } \\
\text { Urgant }\end{array}$ & $\begin{array}{c}\text { Russisches Äquivalent } \\
\text { amerikanischer Late-Night- } \\
\text { Talkshows unter der Moderation } \\
\text { von Ivan Urgant }\end{array}$ \\
\hline
\end{tabular}

line leicht zu finden und frei zugänglich. Der Zugriff auf Talkshows des letzten verbliebenen unabhängigen russischen Fernsehsenders Dožd' ist hingegen leider kostenpflichtig. Da für Lehrpersonen der einfache und freie Zugriff zu Quellenmaterial eine wichtige Rolle spielt, konzentriert sich obige Tabelle auf den Ersten Kanal. Allerdings sollte bei einem Gesamtüberblick über das Fernsehen in Russland im Unterricht unbedingt auf den Sender Dožd' verwiesen werden. Die Lehrperson kann zudem diesen Sender bzw. dessen Webseite nutzen, um eine alternative Meinung und einen kritischen Blick auf die russische Medienwelt zu erhalten. ${ }^{2}$

2 Auf Dožd' gibt es sehr viele schriftliche inhaltliche Kurzzusammenfassungen von Sendungen sowie teilweise auch Transkripte. Für eine Kritik an der Art und Weise, wie mit den Gästen russischer Talkshows auf staatlichen Sendern umgegangen wird, s. https://tinyurl.com/y7ho5kbj [02.12.2017]. 
Das Potenzial der einzelnen in Tab. 1 aufgelisteten Talkshows wird an dieser Stelle genauer erläutert. Für alle vier Talkshows gilt, dass sie lediglich zwischen 30 (Smak, Poka vse doma, Večernij Urgant) und 50 Minuten (Modnyj prigovor) dauern und daher auch in einer Unterrichtsstunde zur Gänze angesehen werden können. Zudem eignen sie sich aufgrund ihrer Thematik sehr gut für die Förderung der interkulturellen Kompetenz sowie der Medienkompetenz, da die SchülerInnen zum einen die Talkshow als „kommunikationsorientierte[s] audiovisuelle[s] Format" (Surkamp 2017: 171) besser kennenlernen, und zum anderen die russische Umsetzung und Besonderheit dieses Formats mit deutschen/österreichischen/amerikanischen Talkshows vergleichen können.

Die problemorientierte Talkshow Modnyj prigovor ist ab dem Anfangsunterricht nicht nur wegen der sehr langsamen Sprechgeschwindigkeit des Moderators, sondern auch aufgrund der für Talkshows typischen repetitiven Elemente einsetzbar. So sind bspw. die einfachen Personenbeschreibungen zu Beginn jeder Sendung immer gleich aufgebaut und die Konzentration liegt auf einer Person, insb. auf ihrem Kleidungsstil. Des Weiteren eignet sich die Show für eine kritische Diskussion über geschlechterspezifische Stereotype und Rollenbilder, da das Aussehen von Frauen (und Männern) im Vordergrund dieser Show steht. Trotz der starken Betonung klassischer Geschlechterrollen versucht Modynj prigovor Toleranz zu vermitteln und das Selbstwertgefühl der TeilnehmerInnen zu stärken. Zudem steht die Konfliktschlichtung ganz oben auf ihrer Agenda, da es schließlich immer darum geht, einen modischen Konflikt zu lösen und Kompromisse zu finden. Auch die Ideologie des Glamours, wie von Caspers \& Scharlaj im vorliegenden Sammelband vorgeschlagen, kann für die Analyse dieser Talkshow herangezogen werden, weil die Show durch ihre Konzentration auf Mode und Schönheit ,Glamour ' - die Vereinigung von Schönheit, Glanz und Charme (vgl. Zvereva 2012: 16) - transportiert. In Verbindung mit den Termini „Glanz“ und "Charme“ und in Hinblick auf den Namen der Show empfiehlt sich außerdem eine detaillierte Analyse der Showkulisse: Diese erinnert an einen Gerichtssaal im Stil eines Catwalks, in dem eine Person, vorgeführt' und beurteilt wird. Eine kritische Betrachtung der Talkshow ist jedoch auf allen Niveaustufen aufgrund ihres augenscheinlich voyeuristischen Charakters notwendig.

Anka Bergmann, Olga Caspers \& Wolfgang Stadler (Hg.)

Didaktik der slawischen Sprachen - Beiträge zum 1. Arbeitskreis in Berlin (12.-14.9.2016)

(C) 2018 innsbruck university press, ISBN 978-3-903187-11-5, DOI 10.15203/3187-11-5 
Modnyj prigovor erweckt also die Illusion, dass das Aschenputtel-Märchen wahr werden kann, wenn sich einfache Leute in der Show in modisch gekleidete und gestylte, Stars' verwandeln. Im Gegensatz dazu beschäftigt sich die Promitalkshow Poka vse doma mit dem Leben und der Karriere prominenter russischer Personen. Am runden Tisch wird im Kreise der Familie in scheinbar ungezwungener Atmosphäre über biographische Ereignisse und die Karriere geplaudert. Das Gespräch wird dabei immer wieder von kurzen Filmsequenzen, Rückblicken und Montagen, die Kindheit, Hochzeit oder Konzertauftritte der Prominenten zeigen, unterbrochen. In diesen kurzen Unterbrechungen befinden sich interessante Artefakte russischer Kultur, wie bspw. Ausschnitte aus bekannten russischen Filmen, Lieder, Musikvideos, wichtige Ereignisse etc. Mithilfe dieser Talkshow lernen die SchülerInnen/Studierenden in 30 Minuten nicht nur eine/n berühmte/n Russen/Russin näher kennen, sondern sie gewinnen auch Einblicke in deren Schaffen und erfahren mehr über deren Beitrag zum russischen kulturellen Leben. Erwähnenswert ist zudem, dass in dieser Talkshow, die sich selbst als Familientalkshow bewirbt, für die Bildung neuer Familien geworben wird. Am Ende der Talkshow verlautet ein Familienmitglied, meistens die Frau, die Ankündigung „U vas budet rebënok“ und danach werden den FernsehzuseherInnen Kinder aus Waisenhäusern präsentiert oder bereits angelaufene Projekte zur Unterstützung von Waisen vorgestellt. Dieses Kuriosum könnte dazu genutzt werden, den Auftrag öffentlich-rechtlicher Fernsehsender in Österreich bzw. Deutschland mit dem Auftrag bzw. Ziel des Pervyj kanal in Russland zu vergleichen und kritisch zu reflektieren.

Eine sich ebenfalls mit Prominenten befassende Talkshow ist die Kochshow Smak, die von Ivan Urgant moderiert wird. Das Besondere an dieser Show wird beim Ansehen des Vorspanns deutlich: Porträts von Puškin, Gogol’ oder Čechov werden mit Bildern von Obst und Gemüse und einem Grimassen schneidenden Ivan Urgant montiert. Russische Schriftsteller zieren auch die Wände der Kochshowküche und wechseln sich von Sendung zu Sendung ab. In dieser Show ist es durchaus nichts Ungewöhnliches, dass der Moderator Gedichte Turgenevs rezitiert oder gemeinsam mit dem Gast ein Lied singt. Das Kochen wird in diesem literarisch-intellektuellen Rahmen zur Nebensache. Für den Unterricht liegt das

Anka Bergmann, Olga Caspers \& Wolfgang Stadler (Hg.)

Didaktik der slawischen Sprachen - Beiträge zum 1. Arbeitskreis in Berlin (12.-14.9.2016)

(C) 2018 innsbruck university press, ISBN 978-3-903187-11-5, DOI 10.15203/3187-11-5 
Potenzial dieser Talkshow neben allen möglichen sprachlichen Aktivitäten rund um das Thema Essen in ihrem literarischen Hintergrund, da mithilfe der Bilder des Vorspanns oder der Analyse der Kücheneinrichtung auf die Porträts der Schriftsteller und ihr literarisches Schaffen eingegangen werden kann. Auch die augenscheinliche männliche Dominanz dieser Kochshow bietet Anlass zur kritischen Diskussion. Für Hör-Seh-Aufgaben eignet sich die gesamte Talkshow aufgrund der schnellen und manchmal undeutlichen Sprache eher für ein höheres Sprachniveau, wobei die kurze Zusammenfassung des gesamten Kochvorgangs am Ende der Sendung aufgrund der Kombination von Bild und Ton sicherlich auch bereits auf Anfängerniveau einsetzbar ist.

Smak als Kombination aus Kunst und Kulinarik ist für die Förderung der interkulturellen Kompetenz besonders wertvoll: Einerseits werden die Lernenden mit fremden Gerichten und Lebensmitteln konfrontiert; andererseits lädt diese Show auch dazu ein, sich mit den Biographien bekannter russischer Schriftsteller auseinanderzusetzen und ihre bis heute zentrale Rolle in der russischen Gesellschaft zu diskutieren. Kochsendungen allgemein sind aufgrund ihrer visuellen, sprachlichen und oft auch schriftlichen Beschreibung der gezeigten Rezepte ideal für den Sprachunterricht. Besonders hervorzuheben ist an dieser Stelle die Kochduellshow Vremja obedat' auf dem Pervyj Kanal, in der durch den Vergleich russischer und internationaler Gerichte die ZuseherInnen die Unterschiede und Besonderheiten der jeweiligen landestypischen Rezepte kennenlernen.

Die vierte Talkshow, die im Rahmen dieses Artikels vorgestellt wird, ist die in Russland v. a. unter jungen Leuten sehr beliebte Late-Night-Talkshow Večernij Urgant. Diese Show ist die russische Adaption amerikanischer Late-Night-Shows und ihr Gastgeber, Ivan Urgant, ist das russische Pendant zu bekannten amerikanischen Moderatoren wie Jimmy Fallon oder David Letterman. Aufgrund dessen bietet sich ein internationaler Vergleich amerikanischer, russischer und deutscher Late-Night-Shows (z. B. Jan Böhmermanns Neo Magazin Royale) sowie eventuell anderer Länder an, um die jeweilige nationale Adaption des Late-Night-Talkshowformats zu untersuchen. Insb. in einer heterogenen Gruppe mit StudentInnen einer anderen L1 als Deutsch oder zur Einbindung der Mehrsprachigkeit im Unterricht ist ein internationaler Vergleich aus kultureller als auch sprachlicher Sicht bereichernd.

Anka Bergmann, Olga Caspers \& Wolfgang Stadler (Hg.)

Didaktik der slawischen Sprachen - Beiträge zum 1. Arbeitskreis in Berlin (12.-14.9.2016)

(C) 2018 innsbruck university press, ISBN 978-3-903187-11-5, DOI 10.15203/3187-11-5 
Im Gegensatz zu den anderen in diesem Artikel erwähnten Talkshows ist die Kombination aus Comedy und Talkshow ein Charakteristikum dieses Fernsehprojekts. Aufgrund ihrer unterschiedlichen Teile - der Anfangsmonolog zu einem aktuellen Thema, ein Talkteil und wechselnde Unterrubriken (Vzgljad snizu, Mimi-mi, Strižëm na Pervom) - können kurze Ausschnitte dieser Talkshow beliebig in den Unterricht eingebaut werden. Allerdings ist diese Late-Night-Talkshow aufgrund des sprachlichen Anspruchs und der Comedy-Elemente eher für den Fortgeschrittenenunterricht (ab B2) zu empfehlen, da bspw. umgangssprachliche Ausdrücke vorkommen, Ivan Urgant ein sehr hohes Sprechtempo hat und teilweise undeutlich spricht. Zudem gehören Witz, Ironie und Wortspiele des Moderators zu den Markenzeichen dieser Show, die damit mehr noch als die anderen Talkshows über die Sprache funktioniert.

\section{Didaktisch-methodische Überlegungen und ein Beispiel für den Unterricht}

\section{Allgemeine Überlegungen}

Wie im Forschungsüberblick gezeigt wurde, sind neben der Authentizität des im Unterricht verwendeten Materials auch die dafür konzipierten Aufgabenstellungen ausschlaggebend für einen authentischen Sprachunterricht. Daher werden in diesem Abschnitt konkrete Vorschläge dafür gemacht, wie die Talkshows oder Sequenzen davon in authentische Aufgabenstellungen eingebettet werden können.

Laut Bechtel \& Roviró (2010: 213) sind Lernaufgaben authentisch, wenn sie die LernerInnen ansprechen, sich mit aktuellen Themen aus ihrer Lebenswelt beschäftigen, nach Kommunikationsformen verlangen, die in der realen Welt vorkommen. Obwohl das Fernsehen generell in der jungen Bevölkerung an Bedeutung verliert, wurden speziell die im vorigen Abschnitt vorgestellten Talkshows ausgewählt, da sie ein breites, und - wie Večernij Urgant - ein junges Publikum ansprechen und im Internet verfügbar sind. Wie bereits erwähnt, ist die Auseinandersetzung mit dem russischen Fernsehen auch im Sprachunterricht 
wichtig und bei der Auswahl neuerer Talkshowsendungen ist sowohl Aktualität als auch Relevanz gegeben.

Um das Fernsehverstehen in den Russischunterricht zu integrieren, bietet sich zunächst eine Analyse des Fernsehprogramms des Pervyj kanal und ein Vergleich mit deutschen/österreichischen Entsprechungen einiger Sendungsnamen bekannter internationaler Formate Wer wird Millionär? (Kto chočet stat' millionerom?), The Voice (Golos), Wheel of Fortune (Pole čudes) sowie russischer Eigenproduktionen (Čelovek i zakon) an. Einerseits wird damit Wortschatz geübt; andererseits können die Lernenden die Fernsehprogramme miteinander vergleichen und etwaige Besonderheiten und Kuriositäten herausarbeiten.

Authentische Aufgabenstellungen lassen sich ebenfalls mit der Förderung der Lernerautonomie verbinden. Diese kann durch eine Kombination aus Präsenzunterricht und privatem Studium zuhause erreicht werden. Jede/r Lernende wählt eine Talkshow oder eine Talkshowsendung aus, sieht sich diese zuhause an und stellt sie anschließend im Unterricht vor (vgl. Thaler 2010: 144). Das Ansehen von Talkshows im privaten Kontext stellt zudem eine authentische Lernsituation dar, weil sie dem echten Fernsehkonsum zuhause entspricht. Alternativ dazu könnten die Lernenden auch eine berühmte Person, die in einer Promitalkshow (z. B. Poka vse doma) vorgestellt wird, auswählen und diese anschließend vor der Klasse oder Gruppe präsentieren. Dadurch wird das kulturelle Wissen über Russland ausgebaut, da in Poka vse doma nicht nur SängerInnen oder SchauspielerInnen zu Gast sind, sondern auch russische Schachspieler, Regisseure, OpernsängerInnen, BalletttänzerInnen oder SportlerInnen, sodass ein breites Themen- und Interessensgebiet abgedeckt wird.

Talkshows können jedoch auch gemeinsam im Unterricht von Lehrpersonen und Lernenden angesehen werden. Als Vorlage dafür, wie diese im Unterricht eingesetzt werden können, dienen die von Thaler (2010: 144) beschriebenen unterschiedlichen Präsentationsmodi für Filme, die ebenso auf Talkshows angewendet werden können. Für Talkshows eignen sich die Block-Präsentation, in der die gesamte Talkshow auf einmal angesehen wird, sowie die SegmentPräsentation, in der lediglich eine Sequenz einer Talkshow gezeigt wird, wie etwa die zusammenfassende Wiederholung des gesamten Rezepts in Smak oder der Vorspann der Talkshow. Ein Vorteil der Segment-Präsentation ist die Konzentra-

Anka Bergmann, Olga Caspers \& Wolfgang Stadler (Hg.)

Didaktik der slawischen Sprachen - Beiträge zum 1. Arbeitskreis in Berlin (12.-14.9.2016)

(C) 2018 innsbruck university press, ISBN 978-3-903187-11-5, DOI 10.15203/3187-11-5 
tion auf eine wichtige Sequenz und die Wiederholbarkeit aufgrund des geringen Zeitbedarfs. In der Möglichkeit der Präsentation von lediglich einer Sequenz liegt die Stärke der Talkshow: Dank ihres Seriencharakters und ihrer Regelmäßigkeit geht beim Ansehen kurzer Sequenzen im Gegensatz zu Filmen, die als Gesamtkunstwerk zu betrachten sind, die Ästhetik einer Talkshow nicht verloren. Weniger geeignet für Talkshows ist allerdings die Intervall-Präsentation, ergo die Aufteilung in einzelne Sequenzen und auf mehrere Unterrichtsstunden, da Talkshowsendungen dafür zu kurz sind und dadurch ihr Unterhaltungswert verloren gehen würde.

\section{Einsatzmöglichkeiten im Unterricht am Beispiel der Talkshow Modnyj prigovor}

Für die Arbeit mit audiovisuellen Medien bieten sich im Allgemeinen nach wie vor die Methoden der Filmdidaktik an, in der die Aufgaben in pre-, while- und post-viewing-activities aufgeteilt werden (vgl. z. B. Caspers \& Scharlaj im vorliegenden Sammelband). Speziell für die TV-Didaktik gibt es jedoch vier eigene Dimensionen, die zu berücksichtigen sind: die semiotische, die sprachliche, die interkulturelle sowie die produktionsorientierte Dimension (vgl. Surkamp 2017: 351f.). Am Beispiel der Sendung ${ }^{3}$ vom 27. November 2017 der Talkshow Modnyj prigovor soll im Folgenden verdeutlicht werden, wie diese im Unterricht auf A2Niveau eingesetzt werden könnte.

Für den Einstieg in die Unterrichtsstunde eignet sich die semiotische Dimension, welche die „Entschlüsselung medialer Texte“ (Surkamp 2017: 351) zum Ziel hat. Dafür bietet sich die Internetseite der Talkshow ${ }^{4}$ auf dem Pervyj kanal an. Darauf befinden sich ein Foto der drei Hauptakteure der Show, der Moderator sowie zwei Modeexpertinnen, und das visuelle Logo (Abb. 1), das von der Lehrperson zunächst verdeckt werden soll. Die Lernenden sollen erraten, was die Personen beruflich machen, ihr Aussehen beschreiben und Vermutungen über das Thema der Show anstellen.

3 Sendung „Delo o tom, kak nočnye dežurstva priveli $k$ dnevnym košmaram“: https://tinyurl.com/yaso23t7 [02.12.2017].

4 Die Internetseite ist online abrufbar: https://tinyurl.com/ybauo2py [02.12.2017].

Anka Bergmann, Olga Caspers \& Wolfgang Stadler (Hg.)

Didaktik der slawischen Sprachen - Beiträge zum 1. Arbeitskreis in Berlin (12.-14.9.2016)

(C) 2018 innsbruck university press, ISBN 978-3-903187-11-5, DOI 10.15203/3187-11-5 


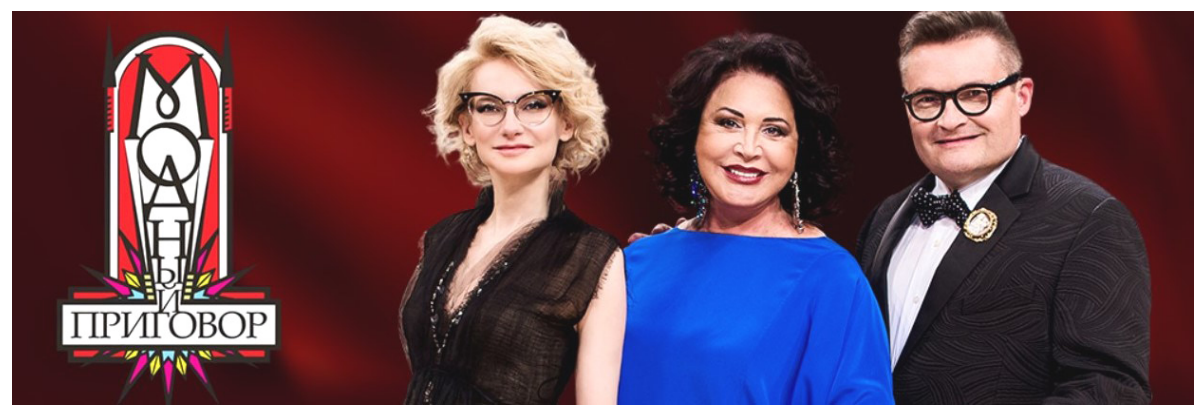

Abbildung 1: Logo von Modnyj prigovor und Foto der ModeratorInnen; Quelle: https://tinyurl.com/ybauo2py [02.12.2017].

Опишите персонажей на фотографии. Кем они работают, что они ^юбят, как они выглядят и какую одежАу они носят? Как вы думаете, какая может быть тематика данного телевизионного жанра?

Danach wird schließlich auch das Logo Modnyj prigovor gezeigt und weitere Vermutungen sollen angestellt werden. Mithilfe eines Wörterbuchs sollen die SchülerInnen eine deutsche Entsprechung für den Namen der Show finden (Modisches Urteil). Im Anschluss daran werden die SchülerInnen in Kleingruppen eingeteilt. Die SchülerInnen sollen selbst die Internetseite der Talkshow öffnen und recherchieren, an welchen Wochentagen, zu welcher Uhrzeit und auf welchem Fernsehsender die Talkshow läuft. Diese Recherche gibt ihnen Aufschluss über das Zielpublikum der Show. Außerdem sollen sie mithilfe der Internetseite und der dort aufscheinenden unterschiedlichen Ausgaben der Show (vypuski) herausfinden, um welches Fernsehformat es sich bei dieser Show handelt (Talkshow).

В какие Ани недели и в какое время Аня проходит это шоу? На каком телеканале оно показывается? Как называется такой формат «шоу» на телевидении?

Danach soll generell auf das Format Talkshow mithilfe eines Brainstormings zur Aktivierung des Vorwissens der SchülerInnen eingegangen werden. Auf A2-Ni- 
veau wird diese Besprechung wahrscheinlich teilweise auch auf Deutsch ablaufen, wobei jedoch die Lehrperson wichtige russische Begriffe im Zusammenhang mit Talkshows auf der Tafel anschreibt. Ein mögliches Tafelbild bzw. auf dem Computer kreiertes Mindmap (Abb. 2) auf A2-Niveau könnte folgendermaßen aussehen:

Dieses zunächst inhaltliche Brainstorming könnte im Anschluss durch den/ die LehrerIn weiter ausgebaut, vertieft und zur Worschatzarbeit genutzt werden, indem die Sammlung von Nomina (Abb. 2) um Verba und Adjektiva ergänzt wird (Abb. 3).

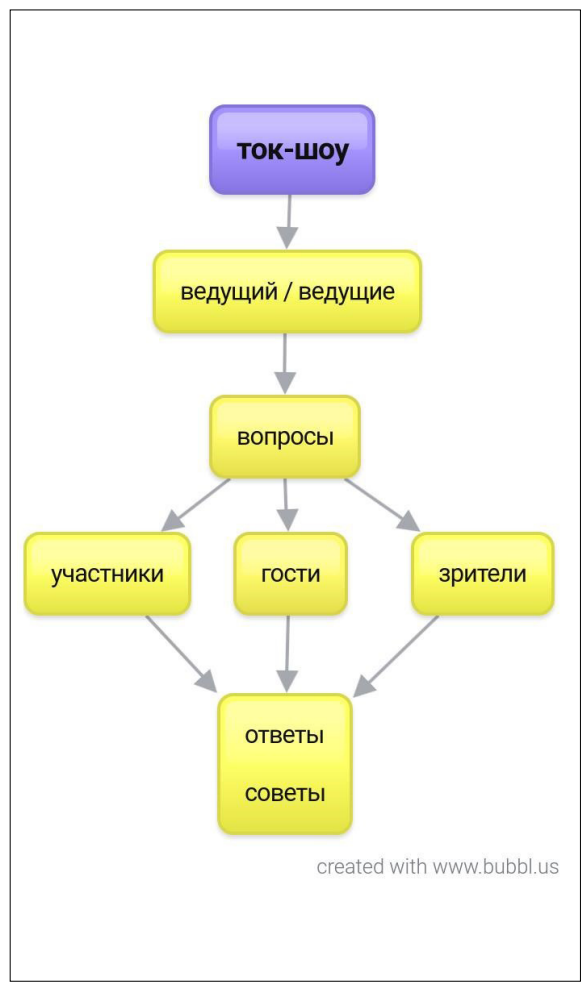

Abbildung 2: Einfaches Mindmap zum Thema Talkshow; Erstellung mithilfe von bubbl.us

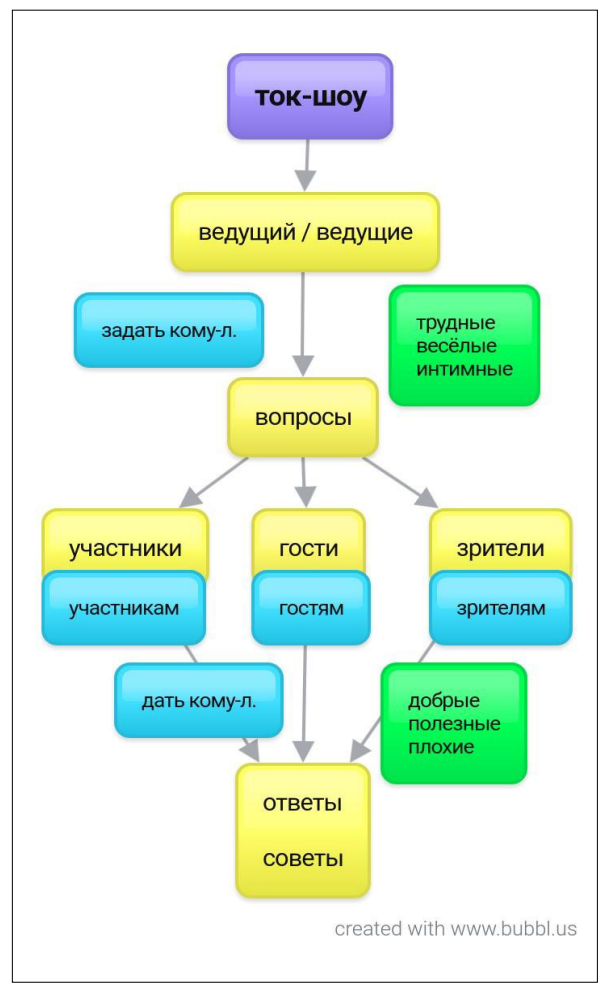

Abbildung 3: Erweitertes Mindmap zum Thema Talkshow; Erstellung mithilfe von bubbl.us 
Danach bietet es sich auf höheren Niveaus an, die kurze Beschreibung zur Akquirierung neuer TeilnehmerInnen der Show zu lesen ${ }^{5}$. Auf A2-Niveau könnte der Text mithilfe der Lehrperson gemeinsam in der Klasse gelesen werden, um das Ziel der Talkshow zu eruieren.

Im Anschluss daran zeigt die Lehrperson die erste Sequenz ${ }^{6}$ (00:00-00:42) der Talkshow, die sich sehr gut für eine Hör-Seh-Verständnisaufgabe auf Anfängerniveau eignet, da die einfachen Angaben (Alter, Name, Beruf, Familienstand, Größe, Gewicht, Kleidungsgröße etc.) mithilfe der Bilder bereits auf diesem Niveau leicht verständlich sind und diese Angaben in manchen Sendungen zusätzlich visuell abgebildet werden. ${ }^{7}$

Danach beginnt die Beschäftigung mit der semiotischen sowie mit der interkulturellen Dimension, welche die „Untersuchung von gesellschaftlichen Selbstwahrnehmungs- und Repräsentationsmustern sowie Geschichte und Mentalitäten einer Kultur“ als auch die Art und Weise, „wie Fernsehen kulturelle Selbst- und Fremdbilder inszeniert und dadurch unsere Vorstellung von Realität beeinflusst“ zum Ziel hat (Surkamp 2017: 352). Dafür sollen die SchülerInnen die Talkshow weiter ansehen und zunächst auf die Showkulisse achten, um den speziellen Aufbau der Show, der an einen Gerichtssaal erinnert, zu ermitteln. Zudem sollen sie Vermutungen darüber anstellen, in welcher Beziehung die Frau und der Mann, die im Zentrum dieser Sendung sind, stehen. Eine geeignete Methode für diese Beobachtungsaufgabe ist silent viewing, weil damit der Fokus auf das Bild gerichtet wird (00:44-1:46).

Как построено это шоу и какие его особенности? Как вы думаете, какое отношение имеют мужчина и женщина в этом ток-шоу Аруг к Аругу? (брат и сестра, муж и жена, друзья, не знаюот друг друга)

5 Zur Projektbeschreibung von Modnyj prigovor: https://tinyurl.com/y7pvabpq [02.12.2017].

6 Sendung „Delo o tom, kak nočnye dežurstva priveli $k$ dnevnym košmaram“: https://tinyurl.com/yaso23t7 [02.12.2017].

7 Eine visuelle Abbildung in einem Dos'e findet sich bspw. zu Beginn dieser Sendung: https://tinyurl.com/yb4dccc7 [02.12.2017].

Anka Bergmann, Olga Caspers \& Wolfgang Stadler (Hg.)

Didaktik der slawischen Sprachen - Beiträge zum 1. Arbeitskreis in Berlin (12.-14.9.2016)

(C) 2018 innsbruck university press, ISBN 978-3-903187-11-5, DOI 10.15203/3187-11-5 
Im Anschluss werden die SchülerInnen in Gruppen eingeteilt, die sich jeweils auf bestimmte Aspekte der Talkshow konzentrieren sollen. Mithilfe dieses sogenannten split viewings soll eine Gruppe auf die Kameraführung und die Schnitte achten, eine zweite Gruppe auf die Frau und ihren Ehemann sowie ihr Auftreten und eine dritte Gruppe auf alle anderen Personen sowie das Licht und den Applaus (01:46-06:45). Im Plenum werden dann die Ergebnisse miteinander verglichen und das zentrale Thema der Show - ein Ehekonflikt aufgrund unterschiedlicher modischer Vorstellungen - gemeinsam herausgearbeitet und kritisch reflektiert.

Тема Аанного ток-шоу - (модный) конфликт межАу супругами. По словам мужа - в паре больше нет романтики, так как стиль и одежАа жены ему не нравятся.

На сколько Аля вас важна внешность (прическа, одежАа, макияж) и внешность Аюбимого человека? Следите Аи вы за модными тенденциями (например, в Инстаграме)?

Das Thema Ehekonflikt ist aus mehreren Gründen für den (schulischen) Unterricht geeignet. Erstens aus interkultureller Sicht, da die beiden Ehepartner der Show erst 27 Jahre alt und bereits einige Jahre verheiratet sind und die SchülerInnen dadurch mit einem kulturellen Unterschied konfrontiert werden: RussInnen heiraten tendenziell früher und in Russland wird generell dem Bild der traditionellen Familie mehr Bedeutung beigemessen als bspw. in Österreich. Zweitens erinnern die Themen dieser Sendung - Mode, Liebe, Eifersucht und Beziehungskonflikt - an bekannte Jugendserien (z. B. Gossip Girl oder Girlboss) und entsprechen damit dem Interessensgebiet vieler SchülerInnen. Darin liegt auch das Potenzial von Modnyj prigovor, da nicht nur die in dieser Talkshow präsentierten Geschlechterklischees thematisiert und reflektiert werden sollen, sondern insb. auch der Konsum- und Selbstdarstellungszwang in Verbindung mit dem Schlankheits- und Schönheitskult, wie er z. B. auf Instagram propagiert wird. Somit schließt die Talkshow thematisch an die Erfahrungs- und Lebenswelt der Lernenden an. Auf höheren Sprachniveaus bietet es sich an, mittels Hörverständnisaufgaben auf Details dieses Ehekonfliktes einzugehen (keine Romantik mehr in der Beziehung, Andeutung einer eventuellen Affäre etc.) und diese herauszu-

Anka Bergmann, Olga Caspers \& Wolfgang Stadler (Hg.)

Didaktik der slawischen Sprachen - Beiträge zum 1. Arbeitskreis in Berlin (12.-14.9.2016)

(C) 2018 innsbruck university press, ISBN 978-3-903187-11-5, DOI 10.15203/3187-11-5 
arbeiten. Das Überthema ,Ehekonflikt' ist jedoch mithilfe der Bildsprache und einzelner Wörter auch auf A2-Niveau verständlich.

Danach sollen die Lernenden mithilfe von Bildern die bis jetzt präsentierten Personen der Talkshow ihren Funktionen zuordnen. Dabei wird noch einmal auf das zuvor besprochene Charakteristikum der Show, deren Setting wie ein Gerichtssaal mit Catwalk aussieht, eingegangen. Zudem wird in der Show mit Gerichtstermini gearbeitet, mit denen die Lernenden bekannt gemacht werden sollen: So gibt es einen Kläger (istec), eine/n VertreterIn der Anklage (obvinitel), eine/n VerteidigerIn (zaščitnik), eine/n Angeklagte/n (obvinjaemyj) sowie einen Leiter der Verhandlung des Modegerichts (veduščij zasedanija modnogo suda). Im Anschluss daran sollen die Lernenden Vermutungen darüber anstellen, was in den nächsten 40 Minuten der Talkshow passieren wird. Außerdem sollen sie sich überlegen, wie sie selbst die Talkshow weitergestalten würden, wenn sie die Möglichkeit dazu hätten:

Как вы думаете, что будет Аальше происходить в этом шоу?

Вы - продюсер этого ток-шоу: Кого вы ещё пригласите на шоу и как оно закончится?

Nach der Präsentation der Vorschläge der Lernenden erläutert die Lehrperson den weiteren Ablauf der Talkshow und fasst die einzelnen Teile unter Präsentation kurzer Ausschnitte zusammen:

- Besprechung ausgewählter Kleidungsstücke der Frau

- Diskussion zwischen den Ehepartnern und Fragen der Verteidigerin sowie der Vertreterin der Anklage bzw. Modeexpertin

- Modnye sovety: Modetipps für alle ZuseherInnen und Erläuterungen der Modeexpertin

- Präsentation der Kleidungsstücke, die der Ehemann für seine Frau ausgewählt hat und Bewertung durch eine unabhängige Modeexpertin

- Präsentation der Kleidungsstücke und Umstyling der Frau durch ModeexpertInnen und Bewertung durch Ehemann und Freunde 
Insgesamt werden sechs verschiedene Outfits für die Frau in der Talkshow vorgestellt. Auf A2-Niveau ist es nicht sinnvoll, die gesamte Talkshow anzusehen, da der lange Talk im Mittelteil der Show sprachlich sehr anspruchsvoll ist. Die anschließenden Modetipps (modnye sovety) sowie die Erläuterungen der Modeexpertin bringen zwar Abwechslung in die Show, stellen für die kritische Auseinandersetzung und das Verständnis jedoch keinen Mehrwert dar. Denkbar ist allerdings, dass sich die Lernenden in Kleingruppen aufteilen und jeweils ein in der Show präsentiertes Outfit auswählen, die Sequenz, in der das Outfit präsentiert wird, ansehen, das Outfit beschreiben, selbst bewerten und dann den anderen Lernenden vorstellen und von ihnen wiederum bewerten lassen. Am Beispiel eines bekannten russischen Sprichwortes bietet sich im Anschluss wiederum eine kritische Reflexion bzw. Sensibilisierung der Lernenden dafür an, dass ein zu rasches Urteil aufgrund des äußeren Erscheinungsbildes einer Person nichts über ihre inneren Werte oder ihre Intelligenz aussagt.

«По одежке встречают, по уму провожают»-известная русская пословица. Что она означает?

Nach diesem interaktiv-reflexiven Teil sollen die Lernenden die ,Verwandlung bzw. das Umstyling der Frau selbst ansehen (39:04-42:02) und dabei auf das Publikum, den Ehemann und die Frau achten, um danach deren Emotionen zu beschreiben. Dafür eigenen sich einfache Phrasen aus der Talkshow (Emy/ ей нравится/нраватся; Он поражен/она поражена; Он/она в восторге) sоwiе Adjektive (потрясаючий, шикарный, гармоничнъй, прекрасныгй).

Abschließend bietet sich wiederum die Integration der interkulturellen Dimension an, da die Lernenden kritisch darüber reflektieren sollen, welches Gesellschaftsbild und welche Geschlechternormen in dieser Show transportiert werden und sie könnten diese mit den Geschlechterrollen in Deutschland/Österreich vergleichen. Mithilfe einfacher Wendungen kann die Beantwortung der folgenden Fragen bereits auf A2-Niveau erfolgen: 
Какую роль играют мужчины/женщины в этом ток-шоу (по словам мужа) и что вы об этом думаете? (11:31-11:45) По вашему мнению, какую роль играют мужчины/женщины в Австрии/в Германии? Что они Аолжны или не Аолжны делать?

(По словам мужа / По моему мнению), мужчина должен / женщина должна готовить, сидеть с детьми, работать, помогать чему-л. чем-л., убирать, стирать, делать покупки, красиво одеваться, иметь хорошее образование ...)

In diesem abschließenden Teil ist es einerseits wichtig, dass die Lehrperson die Problematik der in der Show repräsentierten Geschlechternormen aufgreift, eventuelle Geschlechterrollenstereotype der SchülerInnen aufdeckt und diese mit den Lernenden kritisch reflektiert. Auf A2-Niveau könnte diese kritische Auseinandersetzung mit den Geschlechterstereotypen zusätzlich mithilfe einfacher Behauptungen geschehen, die diskutiert werden sollen (z. В. Женцинь уменот яучше готовить. / Женщины должны заниматься домом. / Аевочки игратот скуклами, а мальчики - смашинками. / Мальчики агрессивнее. / Мальчики больше одарены в математике.) Andererseits sollen die SchülerInnen im Sinne der produktionsorientierten Dimension für die „Problematik der Manipulation durch Fernsehbilder" (Surkamp 2017: 352) sensibilisiert werden, indem die Lehrperson sowohl die Übertreibung und den Show- und Unterhaltungseffekt dieser Talkshow thematisiert als auch auf die Manipulierbarkeit von Bildern bzw. Fotos in sozialen Netzwerken hinweist. Denkbare weitere Aufgaben sind zudem, dass die SchülerInnen die positiven und negativen Aspekte dieser Show sammeln und im Plenum präsentieren sowie diese russische Talkshow mit ähnlichen deutschen Shows (z. B. Dein neuer Style) vergleichen.

Insgesamt gibt es je nach Niveaustufe noch viele weitere Aufgaben, die mithilfe dieser Talkshow möglich sind. Gerade auch Kreativaufgaben, wie die Umformung des Talkshowtextes in andere Textformen (z. B. in lyrische Texte) oder die Produktion einer eigenen Talkshow, bieten auf höheren Niveaustufen vielfältige Einsatzmöglichkeiten. 


\section{Schlussbemerkung}

Talkshows eigenen sich aufgrund ihrer Thematik, ihrer Kürze, Vielfältigkeit und ihrer repetitiven Elemente ideal für den Einsatz im Fremdsprachenunterricht. Obwohl Talkshows nicht das echte Leben repräsentieren, weil ihr Ablauf sowie die Thematik vorgegeben sind, gewähren sie interessante Einblicke in das kulturelle Leben Russlands und sind als Fernsehformat authentisch. Außerdem bringen sie den Lernenden ein Medium näher, das in Russland nach wie vor zentral ist und im Unterricht bisher eher vernachlässigt wurde. Eine Integration von Talkshows in den Unterricht ist auch deshalb lohnend, da diese im Gegensatz zu Filmen den Vorteil haben, dass ihre Ästhetik nicht verloren geht, wenn zu didaktischen Zwecken lediglich einzelne Sequenzen daraus verwendet werden. Zudem sind das Fernsehen und die Analyse von Talkshows für die Förderung der Interkulturalität wertvoll, da sie die Lernenden sowohl zum Vergleich mit der eigenen (Fernseh-)Kultur als auch zu kritischer Diskussion und Reflexion der darin präsentierten Werte und Geschlechterbilder anregen. Insb. die auffallend maskuline Dominanz in den Talkshows sowie die Vermittlung traditioneller Rollenbilder sind äußerst kritisch zu betrachten und müssen im Unterricht thematisiert werden. Darin liegt jedoch auch das Potenzial der Analyse populärer Talkshows, da sie als Ausgangspunkt dazu dienen können, den Blick und das Verständnis zu schulen, wie das Fernsehen und die Medien allgemein unsere Vorstellung von Wirklichkeit manipulieren und beeinflussen.

\section{Literaturverzeichnis}

Abraham, U. (2012). Filme im Deutschunterricht (2., aktual. Aufl.). Seelze: Klett, Kallmeyer.

Al Azri, R. H. \& Al-Rashdi, M. H. (2014). The Effect of Using Authentic Materials in Teaching. International Journal of Scientific \& Technology Research, 3 (10), 249-254.

Arnold, E. (1991). Authenticity Revisited: How Real Is Real? English for Specific Purposes, 10, $237-244$.

Anka Bergmann, Olga Caspers \& Wolfgang Stadler (Hg.)

Didaktik der slawischen Sprachen - Beiträge zum 1. Arbeitskreis in Berlin (12.-14.9.2016)

(C) 2018 innsbruck university press, ISBN 978-3-903187-11-5, DOI 10.15203/3187-11-5 
Bechtel, M. \& Roviró, B. (2010). Authentizität von Lernaufgaben im kompetenzorientierten Französisch- und Spanischunterricht. In M. Frings \& E. Leitzke-Ungerer (Hrsg.), Authentizität im Unterricht romanischer Sprachen (207-229). Stuttgart: ibidem.

Bertram, A. (2011). Tema Molodëž’: stiljagi i neformaly. Material für die SEK II, Niveau C1. Praxis Fremdsprachenunterricht Russisch, 4, 7-9.

Binder, E. \& Kaltseis, M. (2016a). «Rusalka» - Großstadtmärchen und Geschichte des Erwachsenwerdens. Praxis Fremdsprachenunterricht Russisch, 5, 7-9.

Binder, E. \& Kaltseis, M.(2016b). «Pervye fil'my» - Kurzfilme des Moskauer VGIK. Praxis Fremdsprachenunterricht Russisch, 6, 7-9.

Brook, J. (2011). The Affordances of YouTube for Language Learning and Teaching. Hawaii Pacific University TESOL Working Paper Series, 9 (1, 2), 37-56.

Creeber, G. (2015) (Hrsg.). The Television Genre Book (3. Auf.). London u. a.: Palgrave Macmillan u. a.

Crossley, S., Louwerse, M., Maccarthy, P. \& Mcnamara, D. (2007). A Linguistic Analysis of Simplified and Authentic Texts. The Modern Language Journal, 91, 15-30.

Engel, C. \& Bacher, S. (2011). Moskau in der Sommerhitze. Zwei Spielfilme. Praxis Fremdsprachenunterricht Russisch, 3, 4-6.

GeR=Trim, J., North, B. \& Coste, D. (Hrsg.) (2001). Gemeinsamer europäischer Referenzrahmen für Sprachen: Lernen, lehren, beurteilen. Berlin: Langenscheidt.

García, M. (2016). Filme und interkulturelles Sehverstehen - ein Vorschlag für den Spanischunterricht. In C. Michler \& D. Reimann (Hrsg.), Sehverstehen im Fremdsprachenunterricht (339-351). Tübingen: Narr.

Henseler, R., Möller, S. \& Surkamp, C. (2011). Filme im Englischunterricht. Grundlagen, Methoden, Genres. Seelze: Klett, Kallmeyer.

Herron, C., York, H., Corrie, C. \& Cole, S. (2006). A Comparison Study of the Effects of a Story Based Video Instructional Package versus a Text-Based Instructional Package IntermediateLevel in the Foreign Language Classroom. CALICO Journal, 23 (2), 281-307.

Hutchings, S. \& Rulyova, N. (2009). Television and Culture in Putin's Russia. Abingdon, New York: Routledge.

Kaltseis, M. (2015). «Legenda № 17». Ein Film über einen der berühmtesten sowjetischen Eishockeyspieler. Praxis Fremdsprachenunterricht Russisch, 2, 10-12.

Lay, T. (2009). Eine empirisch quantitative Erhebung zur didaktisch-methodischen Implementierung filmspezifischer Arbeit im universitären Deutschstudium Taiwans. Zeitschrift für interkulturellen Fremdsprachenunterricht, 14 (1), 107-153. 
Laschet, R. (2008). «Piter FM» - Film für den Russisch-Unterricht. Praktika, 2, 6-18.

Lommel, S. V., Laenen, A. \& Ydewalle d', G. (2006). Foreign-Grammar Acquisition While Watching Subtitled Television Programmes. British Journal of Educational Psychology, 76, 243-258. Lütge, C. (2012). Mit Filmen Englisch unterrichten. Berlin: Cornelsen.

Mac, A. (2011). Zum Einsatz von authentischem Quellenmaterial im Fremdsprachenunterricht am Beispiel von Fernsehnachrichten. Glottodidactica XXXVII, 73-84.

Mikos, L. (1994). Fernsehen im Erleben der Zuschauer: vom lustvollen Umgang mit einem populären Medium. Berlin, München: Quintessenz.

Mishan, F. (2005). Designing Authenticity into Language Learning Materials. Bristol, Portland: Intellect Books.

Nadchuk, E. \& Behr, U. (2012). Maša i Medved': Raz, dva, tri - ëločka gori! Trickfilm im Anfangsunterricht. Praxis Fremdsprachenunterricht Russisch, 6, 9-11.

Nosonovič, E. V. \& Mil’rud, R. P. (1999). Parametry autentičnogo učebnogo teksta. Inostrannye jazyki v škole, 1, 1-14.

Plake, K. (1999). Talkshows. Die Industrialisierung der Kommunikation. Darmstadt: Primus.

Roberts, C. \& Cooke, M. (2009). Authenticity in the Adult ESOL Classroom and Beyond. Tesol Quarterly, 43 (4), 620-642.

Rokni, A. S. J. \& Ataee, J. A. (2014). Movies in EFL Classrooms: With or Without Subtitles. The Dawn Journal, 3 (1), 715-726.

Satzinger, C. (2016). Mit Karambolage sehen(d) verstehen. In C. Michler \& D. Reimann (Hrsg.), Sehverstehen im Fremdsprachenunterricht (244-262). Tübingen: Narr.

Scherpinski, A. (2014). Potenzial audiovisueller Texte für das Fremdsprachenlernen - eine vergleichende quantitative Untersuchung mit koreanischen Germanistikstudierenden zu Verstehensleistungen bei auditiver und audiovisueller Textrezeption. Zeitschrift für Interkulturellen Fremdsprachenunterricht [Online], 19 (2), 171-186.

Semeria, S. (2002). Die Daytime Talkshow. Zur Erfindung eines Genres in den USA und dessen Adaption in Deutschland. In J. Tenscher \& C. Schicha (Hrsg.), Talk auf allen Kanälen (161180). Wiesbaden: Westdeutscher Verlag.

Sherman, J. (2006). Using Authentic Video in the Language Classroom (Reprint). Cambridge u. a.: Cambridge University Press.

Surkamp, C. (2017) (Hrsg.). Metzler Lexikon Fremdsprachendidaktik (2., aktual. u. erw. Aufl.). Stuttgart: J. B. Metzler.

Tenscher, J. \& Schicha, C. (2002) (Hrsg.). Talk aufallen Kanälen. Wiesbaden: Westdeutscher Verlag.

Anka Bergmann, Olga Caspers \& Wolfgang Stadler (Hg.)

Didaktik der slawischen Sprachen - Beiträge zum 1. Arbeitskreis in Berlin (12.-14.9.2016)

(C) 2018 innsbruck university press, ISBN 978-3-903187-11-5, DOI 10.15203/3187-11-5 
Thaler, E. (2007). Schulung des Hör-Seh-Verstehens. Praxis Fremdsprachenunterricht, 4, 12-17.

Thaler, E. (2010). Filmdidaktik. In W. Hallet \& F. G. Königs (Hrsg.), Handbuch Fremdsprachendidaktik (142-146). Seelze-Velber: Klett, Kallmeyer.

Thaler, E. (2014). Teaching English with Films. Paderborn: Schöningh.

Thaler, E. (2017). Authentizität. Praxis Fremdsprachenunterricht, 2, 16.

Vakal, E. (2005). Trickfilme im Russischunterricht. Čeburaška i krokodil Gena. Praxis Fremdsprachenunterricht, 6, 49-54.

Volkov, D. \& Gončarov, S. (2017). Rossijskij Medialandšaft: osnovnye tendencii ispol'zovanija SMI - 2017. Verfügbar unter: https://tinyurl.com/ycbvuft7 [02.12.2017].

Zühlke, M. (2007). Arbeit mit Dokumentarfilmen. Sergej Loznitsas «Pejzaž» (2003). Praxis Fremdsprachenunterricht, 6, 49-54.

Zvereva, V. V. (2012). "Nastojaščaja žizn'» v televizore. Issledovanija sovremennoj mediakul'tury. Moskva: RGGU. 